Abstract

\title{
Dynamical Systems with Spatiotemporal Periodicities through the Symmetries ${ }^{+}$
}

\author{
Niurka R. Quintero 1,2,*, Jesús Casado-Pascual 3, Renato Alvarez-Nodarse ${ }^{4}$ and José A. Cuesta 5,6 \\ 1 Departamento de Física Aplicada I, E.P.S., Universidad de Sevilla, Virgen de Africa 7, 41011 Sevilla, Spain \\ 2 Instituto Carlos I de Física Teórica y Computacional, Universidad de Granada, 18071 Granada, Spain \\ 3 Física Teórica, Universidad de Sevilla, Apartado de Correos 1065, 41080 Sevilla, Spain; jcasado@us.es \\ 4 IMUS and Departamento de Análisis Matemático, Universidad de Sevilla, Apdo 1160, \\ 41080 Sevilla, Spain; ran@us.es \\ 5 Grupo Interdisciplinar de Sistemas Complejos (GISC), Departamento de Matemáticas, \\ Universidad Carlos III de Madrid, Avda. de la Universidad 30, 28911 Leganés, Spain; \\ cuesta.jose.a@gmail.com \\ 6 Instituto de Biocomputación y Física de Sistemas Complejos (BIFI), Universidad de Zaragoza, \\ 50009 Zaragoza, Spain \\ * Correspondence: niurka@us.es \\ + Presented at Symmetry 2017-The First International Conference on Symmetry, Barcelona, Spain, \\ 16-18 October 2017.
}

Published: 3 January 2018

Dynamical systems often contain oscillatory forces or depend on periodic potentials. Time or space periodicity is reflected in the properties of these systems through a dependence on the parameters of their periodic terms [1,2]. In this talk, it is shown that simple symmetry considerations determine how their properties depend functionally on the amplitudes and the phases of the periodic terms, regardless of whether they are classical or quantum, stochastic or deterministic, dissipative or nondissipative [3]. This formalism is applied to find the functional dependence of the expectation value of the momentum of a Bose-Einstein condensate, described by the Gross-Pitaewskii equation [4,5], when it is exposed to a bi-harmonic potential whose amplitude is periodically modulated in time. It is shown that, by using this formalism, a small set of measurements is enough to obtain the functional form for a wide range of parameters.

Acknowledgments: We acknowledge financial support from the MINECO of Spain through FIS2014-54497-P (N.R.Q.) and MTM2015-65888-C4-1-P (R.A.N.).

\section{References}

1. Cuesta, J.A.; Quintero, N.R.; Alvarez-Nodarse, R. Time-shift invariance determines the functional shape of the current in dissipative rocking ratchets. Phys. Rev. X 2013, 3, 041014.

2. Quintero, N.R.; Cuesta, J.; Alvarez-Nodarse, R. Symmetries shape the current in ratchets induced by a biharmonic driving force. Phys. Rev. E 2010, 81, 030102.

3. Casado-Pascual, J.; Cuesta, J.A.; Quintero, N.R.; Alvarez-Nodarse, R. General approach for dealing with dynamical systems with spatiotemporal periodicities. Phys. Rev. E 2015, 91, 022905.

4. Dalfovo, F.; Giorgini, S.; Pitaevskii, L.P.; Stringari, S. Theory of Bose-Einstein condensation in trapped gases. arXiv 1998, arXiv:cond-mat/9806038.

5. Pitaevskii, L.P.; Stringari, S. Bose-Einstein Condensation; Oxford University Press: New York, NY, USA, 2003.

(C) 2018 by the authors. Licensee MDPI, Basel, Switzerland. This article is an open access article distributed under the terms and conditions of the Creative Commons Attribution (CC BY) license (http://creativecommons.org/licenses/by/4.0/). 